\title{
Baryon-antibaryon pair production in strong meson fields*
}

\author{
I N Mishustin†, L M Satarov†, J Schaffnerł̣, H Stöckerł and W Greinerł \\ $\dagger$ The Kurchatov Institute, Moscow 123182, Russia \\ $\ddagger$ Institut für Theoretische Physik, J W Goethe Universität, D-6000 Frankfurt am Main, \\ Federal Republic of Germany
}

Received 10 June 1993

\begin{abstract}
According to the relativistic mean-field model, strong meson fields exist in normal nuclei. Even stronger meson fields may be generated in highly compressed and excited hadronic matter formed in relativistic heavy-ion collisions. The attractive interaction of baryons with a scalar field results in a significant reduction of the energy gap in the Dirac spectrum. The interaction with a vector field generated by baryon-rich medium is repulsive for baryons and attractive for antibaryons. At high enough densities, the minimal energy of antibaryons may become lower then $-m_{\mathrm{B}}$, where $m_{\mathrm{B}}$ is the baryon mass in vacuum. Such a system becomes unstable with respect to the spontaneous creation of baryon-antibaryon pairs. This phenomenon is estimated to occur at densities exceeding the normal nuclear density by factor 37 , depending on the coupling constants. Spontaneous as well as induced pair creation will modify drastically both the antiproton and antihyperon production in relativistic heavy-ion collision. This could have a serious impact on recent proposal to use enhanced antihyperon production as an unambiguous signal for the quark-gluon plasma.
\end{abstract}

\section{Introduction}

In recent years the relativistic quantum hadrodynamics (QHD) model and, in particular, relativistic mean-field model (RMFM) have been widely used for the description of nuclear matter, finite nuclei, and nuclear dynamics (for reviews see $[1,2]$ ). This model is based on the effective Lagrangian of baryons interacting with mean meson fields. At present the limits of applicability of this model, especially far from the nuclear ground state, are unclear. The mean-field approximation should work better at higher densities, when many particles are present in spatial regions characterized by the strong interaction scale of the order of $1 \mathrm{fm}$. But at such densities one can expect corrections due to the finite size and quark-gluon structure of hadrons. The lattice gauge models based on the QCD Lagrangian predict that at an energy density of the order of a few $\mathrm{GeV} \mathrm{fm}^{-3}$ hadronic matter undergoes a phase transition from the baryon-meson phase to the quark-gluon plasma. Unfortunately, the confinement properties of QCD are not understood yet and a quantitaive description of strong interactions in terms of quarks and gluons is rather uncertain far from the asymptotically free region. The most fruitful way to deal with this 'non-perturbative' situation, in our opinion, would be to approach the problem from both sides, i.e. to develop the $\mathrm{QHD}$ - and QCD-motivated models and to compare their predictions with the experimental data. One might expect that in the transition region these models would describe the same observable phenomena, but just in different languages.

* This work has been funded in part by the Bundesministerium für Forschung und Technologie. 
For instance, Theis et al [3] demonstrated that the RMFM exhibits a sudden change in thermodynamical behaviour around $T_{\mathrm{c}} \approx 200 \mathrm{MeV}$ at zero baryon density, in analogy to quark-gluon deconfinement in lattice QCD. In addition, the RMFM predicts a strong reduction of baryon masses. This is similar to the chiral symmetry restoration observed in lattice QCD. High abundance of low-mass baryon-antibaryon pairs at temperatures $\sim T_{c}$ may result in a large enhancement of antiproton and antihyperon yields in relativistic heavy-ion collision. This purely hadronic scenario can explain the recent observation of enhanced $\bar{\Lambda}, \bar{\Sigma}$ production in relativistic heavy-ion collision $[4,5]$. A similar enhancement had been proposed earlier [6] as a potential candidate for a quark-gluon plasma signal. Let us keep this in mind when we use below the RMFM to describe phenomena which may occur far from the nuclear ground state.

Until recentiy only positive energy baryon states (valence baryons) were considered explicitly in applications of the RMFM. But probably the most interesting features of the RMFM are connected with the existence of negative energy baryon states (Dirac sea). Below it is demonstrated that the RMFM predicts a substantial reduction of the baryon effective masses and strongly attractive potentials for antibaryons already at normal nuclear density. This effects become even more pronounced at the higher baryon densities which may be reached in the course of intermediate and high-energy heavy-ion collisions. A strong space and time variation of the background meson fields, as well as high thermal excitation, may lead in this case to the considerable enhancement of the baryon-antibaryon pair production, as compared with predictions of simple models disregarding in-medium effects. These phenomena were first considered in our earlier publications [8-10]. In the present paper we investigate the collective mechanism of antibaryon production in more details and discuss the possible observable effects in relativistic heavy-ion collisions.

The paper is organized as follows. In section 2 we briefly present the model Lagrangian and corresponding equations of motion in the mean-field approximation. In section 3 we discuss the single-particle energies of baryons and antibaryons in mean meson fields generated by the dense baryon-rich medium. In particular, the strongly attractive potentials acting on the antibaryons are emphasized. In section 4 we formulate the critical conditions for spontaneous baryon-antibaryon pair creation and calculate the corresponding critical parameters. The spontaneous pair-production rate in relativistic heavy-ion collisions is estimated in section 5, where possible observable effects associated with the critical phenomena are also discussed. Our conclusions and prospects for future studies are given in section 6 . The reflection coefficient for the combined scalar and vector potentials is derived in the appendix.

\section{Effective Lagrangian}

Below we use the effective Lagrangian [1] containing baryon $\left(\Psi_{B}\right)$, scalar-meson $(\sigma)$ and vector-meson $\left(\omega^{\mu}\right)$ fieldsț:

$$
\begin{aligned}
\mathcal{L}=\sum_{\mathrm{B}} \bar{\Psi}_{B}\left[\mathrm{i} \gamma^{\mu}\left(\partial_{\mu}+\mathrm{i} g_{\mathrm{V}_{B}} \omega_{\mu}\right)-\left(m_{\mathrm{B}}-g_{\mathrm{S}_{B}} \sigma\right)\right] \Psi_{B} \\
\quad-\frac{1}{4} F_{\mu \nu} F^{\mu \nu}+\frac{1}{2} m_{\omega}^{2} \omega_{\mu} \omega^{\mu}+\frac{1}{2} \partial_{\mu} \sigma \partial^{\mu} \sigma-V(\sigma)
\end{aligned}
$$

where the sum runs over the baryon species $B=\mathrm{N}, \Delta, \Lambda, \ldots$,

$$
F_{\mu \nu}=\partial_{\mu} \omega_{\nu}-\partial_{\nu} \omega_{\mu}
$$

† Units with $\hat{h}=c=1$ are used throughout the paper. 
is the vector field strength tensor and

$$
V(\sigma)=\frac{1}{2} m_{\sigma} \sigma^{2}+\frac{b}{3} \sigma^{3}+\frac{c}{4} \sigma^{4}
$$

is the scalar feld potential including self-interaction terms [11]. Here $m_{\mathrm{B}}, m_{\sigma}, m_{\omega}$ are vacuum masses of the corresponding fields, $g_{S B}, g_{\mathrm{V} B}, b$ and $c$ are the coupling constants. The corresponding equations of motion are

$$
\begin{aligned}
& {\left[\mathrm{i} \gamma^{\mu}\left(\partial_{\mu}+\mathrm{i} g_{\vee B} \omega_{\mu}\right)-\left(m_{\mathrm{B}}-g_{S_{B} \sigma} \sigma\right)\right] \Psi_{B}=0} \\
& \partial_{\nu} \partial^{\nu} \sigma+V^{\prime}(\sigma)=\sum_{\mathrm{B}} g_{S B} \rho_{\mathrm{S}}^{(B)} \\
& \left(\partial_{\nu} \partial^{\nu}+m_{\omega}^{2}\right) \omega_{\mu}-\partial_{\mu}\left(\partial_{\nu} \omega^{\nu}\right)=\sum_{\mathrm{B}} g_{\vee B} j_{\mu}^{(B)} .
\end{aligned}
$$

Here $\rho_{\mathrm{S}}^{(B)}=\left\langle\bar{\Psi}_{B} \Psi_{B}\right\rangle$ and $j_{\mu}^{(B)}=\left\langle\bar{\Psi}_{B} \gamma_{\mu} \Psi_{B}\right\rangle$ are, respectively, the scalar density and the 4-current associated with the baryon species $B$. Below we consider the isotropic system at rest. In this case $\omega_{\mu}=\left(\omega_{0}, \mathbf{0}\right)$ and $j_{\mu}^{(B)}=\left(\rho_{\mathrm{V}}^{(B)}, \mathbf{0}\right)$, where $\rho_{\mathrm{V}}^{(B)}=\left\langle\Psi_{B}^{+} \Psi_{B}\right\rangle$ is the vector density of the baryon species $B$. In the mean-feld approximation the meson field operators are replaced by their expectation values. The total scalar and vector densities are given by

$$
\rho_{\mathrm{S}}=\sum_{\mathrm{B}} \rho_{\mathrm{S}}^{(B)} \quad \rho_{\mathrm{V}}=\sum_{\mathrm{B}} \rho_{\mathrm{V}}^{(B)}
$$

In the case of infinite nuclear matter at zero temperature (without hyperons) the model has four adjustable parameters

$$
\begin{array}{ll}
C_{\mathrm{S}}=g_{\mathrm{SN}} \frac{m_{\mathrm{N}}}{m_{\sigma}} & C_{\mathrm{V}}=g_{\mathrm{VN}} \frac{m_{\mathrm{N}}}{m_{\omega}} \\
C_{3}=\frac{b}{m_{\mathrm{N}} g_{\mathrm{SN}}^{3}} & C_{4}=\frac{c}{g_{\mathrm{SN}}^{4}}
\end{array}
$$

where $m_{\mathrm{N}}=938 \mathrm{MeV}$ is the vacuum nucleon mass. These parameters may be fixed by the binding energy $\epsilon_{b}$, baryon density $\rho_{0}$, effective mass $m_{\mathrm{N}}^{*}$ and incompressibility modulus $K$ of isospin-symmetric nuclear matter in equilibrium. The simplest realization of the model $\left(C_{3}=C_{4}=0\right)$ leads to an effective mass which is too low $\left(m_{\mathrm{N}}^{*}=0.56 m_{\mathrm{N}}\right)$ and an incompressibility modulus which is too high $(K \approx 540 \mathrm{MeV})$ [1]. One can get more realistic values of $m_{\mathrm{N}}^{*}$ and $K$ by introducing self-interaction terms in $V(\sigma)$ [11]. If the parameters of the model are fixed by known nuclear matter properties, then the effective masses and mean potentials of baryons as well as antibaryons may be unambiguously calculated on the basis of the RMFM.

\section{The baryon and antibaryon energies in mean meson fields}

Let us consider now a finite region of space occupied by nuclear matter and characterized by a spatial dimension $R$. For sufficiently large $R$ the single particle states can be classified by the 3-momentum $\boldsymbol{p}$ and the internal baryon wavefunctions may be well approximated by 
plane waves. In the static case, the Dirac equation for baryons (3) leads to the following single-particle energy spectrum:

$$
E^{ \pm}(\boldsymbol{p})=U_{\mathrm{V}_{B}} \pm \sqrt{\boldsymbol{p}^{2}+m_{\mathrm{B}}^{* 2}}
$$

where

$$
m_{\mathrm{B}}^{*}=m_{\mathrm{B}}-U_{\mathrm{SB}}
$$

is the effective baryon mass and

$$
\begin{aligned}
& U_{\mathrm{SB}}=g_{S B} \sigma \\
& U_{\mathrm{V} B}=g_{\mathrm{V} B} \omega_{0}
\end{aligned}
$$

are the effective scalar and vector potentials generated by the mean meson fields. These fields are determined selfconsistently from the equations of motion (5), (6) by expressing them in terms of $\rho_{\mathrm{S}}$ and $\rho_{\mathrm{v}}$. Here we consider the nucleonic system at zero temperature. Then all states with $p<p_{\mathrm{F}}$ are occupied, where $p_{\mathrm{F}}$ is the nuclear Fermi momentum in the inner part of the system. The vector and scalar densities are expressed as

$$
\begin{aligned}
& \rho_{\mathrm{V}}=v_{\mathrm{N}} \int_{p \leqslant p \mathrm{~F}} \frac{d^{3} p}{(2 \pi)^{3}}=\frac{\nu_{\mathrm{N}} p_{\mathrm{F}}^{3}}{6 \pi^{2}} \\
& \rho_{\mathrm{S}}=v_{\mathrm{N}} \int_{p \leqslant p_{\mathrm{F}}} \frac{d^{3} p}{(2 \pi)^{3}} \frac{m_{\mathrm{N}}^{*}}{\sqrt{p^{2}+m_{\mathrm{N}}^{* 2}}}=\frac{\nu_{\mathrm{N}} m_{\mathrm{N}}^{*} p_{\mathrm{F}}^{2}}{4 \pi^{2}} \Phi\left(\frac{m_{\mathrm{N}}^{*}}{p_{\mathrm{F}}}\right)
\end{aligned}
$$

where

$$
\Phi(x)=\sqrt{1+x^{2}}-\frac{x^{2}}{2} \ln \left(\frac{\sqrt{1+x^{2}}+1}{\sqrt{1+x^{2}}-1}\right)
$$

and $\nu_{B}$ is the spin-isospin degeneracy factor of baryon $B$ (for nucleons $\nu_{\mathrm{N}}=4$ ). It has been demonstrated that the RMFM based on the nonlinear Lagrangian (1) reproduces well the main properties of atomic nuclei $[1,2]$. The qualitative picture is as follows: the baryons induce meson fields which in turn generate the selfconsistent potentials for baryons. It is important to point out that the relativistic Dirac equation (4) describes simultaneously baryons (nucleons) with an energy $E_{B}(p)=E^{+}(p)$ and antibaryons (antinucleons) with an energy $E_{\vec{B}}(p)=-E^{-}(-p)$. The corresponding mean potentials acting on the baryons and the antibaryons at $p=0$ are respectively

$$
\begin{aligned}
& U_{B}=E_{B}(0)-m_{\mathrm{B}}=U_{\mathrm{V} B}-U_{\mathrm{S} B} \\
& U_{\bar{B}}=E_{\bar{B}}(0)-m_{\bar{B}}=-U_{\mathrm{V} B}-U_{\mathrm{S} B} .
\end{aligned}
$$

Note that the mean potential induced by the vector field is repulsive for the baryons, while it is attractive for the antibaryons. This is a consequence of the negative $G$-parity of $\omega$-meson.

To calculate the potentiais one should solve the equations of motion for the meson fields. Dropping the derivative terms in (5), (6), which is a good approximation for the inner part of the system, one obtains the algebraic equations, which may be solved easily. Using (10) 
and (13) it is convenient to rewrite the equation for $\sigma$ in terms of the nucleon effective mass as a function of the Fermi momentum:

$$
C_{\mathrm{S}}^{-2}\left(1-\frac{m_{\mathrm{N}}^{*}}{m_{\mathrm{N}}}\right)+C_{3}\left(1-\frac{m_{\mathrm{N}}^{*}}{m_{\mathrm{N}}}\right)^{2}+C_{4}\left(1-\frac{m_{\mathrm{N}}^{*}}{m_{\mathrm{N}}}\right)^{3}=\frac{\nu_{\mathrm{N}}}{4 \pi^{2}} \frac{p_{\mathrm{F}}^{2} m_{\mathrm{N}}^{*}}{m_{\mathrm{N}}^{3}} \Phi\left(\frac{m_{\mathrm{N}}^{*}}{p_{\mathrm{F}}}\right)
$$

where $\Phi(x)$ is defined in (14). At a reasonable choice of the parameters $b$ and $c$ in the nonlinear potential (3) the baryon effective mass (10) decreases gradually and tends to zero at high baryon density and temperature. A few examples are shown in figure 1. The asymptotic value of the nucleon effective mass may differ from zero in a multicomponent system of nucleons, baryonic resonances and hyperons. Such a situation may be realized at high temperatures. Formally speaking, at some choices of model parameters the function $m_{\mathrm{N}}^{*}\left(\rho_{\mathrm{V}}\right)$ may even cross zero and become negative. But this does not at all mean that the nucleon effective mass would be negative. According to (9) and the discussion above, in this case one should adopt the mass $\left|m_{\mathrm{N}}^{*}\right|$ for the nucleons and antinucleons. In the cases considered below we never get a negative $m_{\mathrm{N}}^{*}$.

\section{Effective mass in the RMF model}

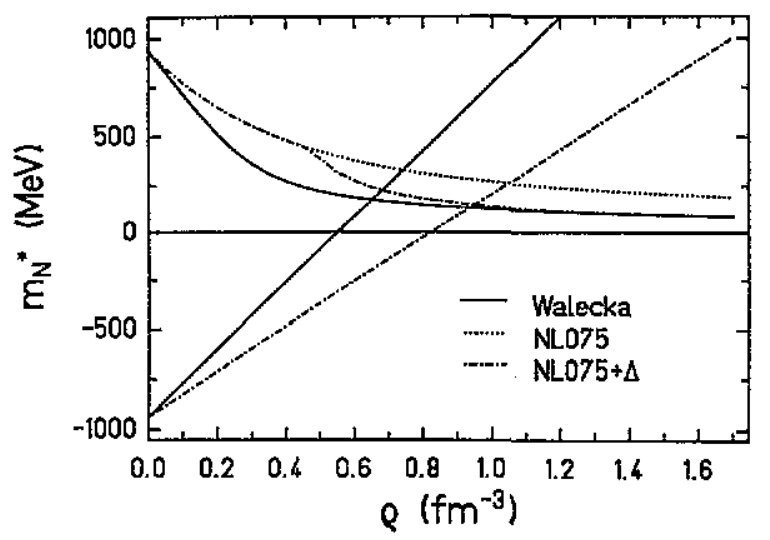

Figure 1. The critical point in compressed nuclear matter is determined by the crossing of the two curves starting at $m_{\mathrm{N}}$ and $-m_{\mathrm{N}}$. The case for the Walecka parameter set (solid lines) is compared with that for a set fitted to finite nuclei (dotted lines). The inclusion of the $\Delta$-isobars (dash-dotted lines) can diminish the critical density. In this calculation the ratio of the $\Delta$ and the nucleon coupling constant is $\mathbf{1 . 3 1}$ for the scalar and 1.0 for the vector field.

For the zero component of the vector field we get, from (6),

$$
\omega_{0}=\frac{g_{\mathrm{VN}}}{m_{\omega}^{2}} \rho_{\mathrm{v}} .
$$

At the normal nuclear density, $\rho_{\mathrm{v}}=\rho_{0}=0.15 \mathrm{fm}^{-3}$, the vector and scalar potentials predicted by the RMFM are rather large, of the order of a few hundred MeV. For nucleons they nearly cancel each other, which results in the observed shallow potential well of about $60 \mathrm{MeV}$. However, for antinucleons a much deeper potential is predicted on the basis of the $G$-parity transformation. For instance, in the original Walecka model $\left(C_{3}=C_{4}=0\right)$ a potential depth of about $700 \mathrm{MeV}$ deep is predicted. In more realistic nonlinear versions of 
the RMFM [11] the antinucleon potentials are usually smaller by a factor $2-3$. The energy levels of nucleons and antinucleons in a spherical nucleus of normal density are shown schematically in figure 2 . It is seen that the gap between upper and lower continuum states, being $2 m_{\mathrm{N}}^{*}$, is substantially reduced inside the nucleus as compared with the vacuum. In the figure one can also see the shallow nucleon potential (with respect to $m_{N}$ ) and the deep antinucleon potential (with respect to $-m_{\mathrm{N}}$ ).

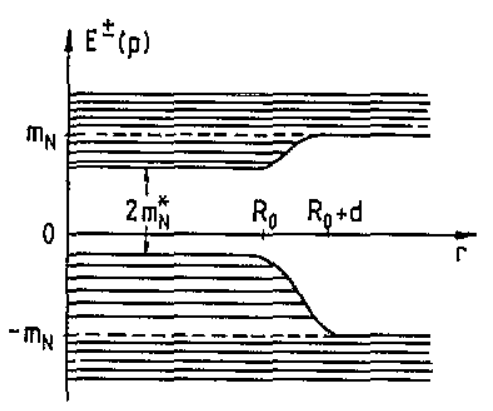

Figure 2. Energy levels of nucleons and antinucleons in a normal nucleus of radius $R_{0}$.

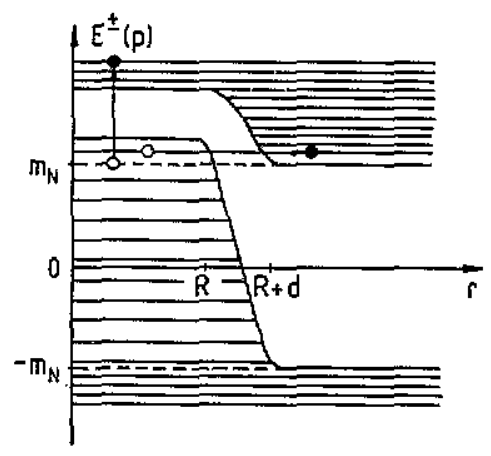

Figure 3. Energy levels of nucleons and antinucleons inside a domain of compressed nuclear matter. Vertical and horizontal arrows correspond to the induced and spontaneous pair-production processes, respectively.

Arguments in favour of a reduced gap and a strongly attractive interaction of antinucleons inside the nucleus were put forward already in 1956 [13]. Later the strong attraction in the nucleon-antinucleon system was emphasized as a possible mechanism for $N \bar{N}$ bound states [14]. The importance of the reduction of the energy gap between baryon and antibaryon states in nuclei was also discussed in [15].

What is known about the antinucleon-nucleus interaction from experiment? Highprecision data are available now at LEAR for antiproton energies of the order of $100 \mathrm{MeV}$. At such energies the antiproton-nucleus interaction is dominated by the large annihilation cross section, i.e. antinucleons can not penetrate deeply into the nuclear interior due to the strong absorption at the surface In contrast, the nucleons can traverse the whole nucleus. From the optical-potential fits of the elastic scattering data $[16,17]$ it is known that the strong absorbtion radius is about $20-30 \%$ larger than the RMS nuclear radius. The real part of the optical potential at this radius is very small, about $-5 \mathrm{MeV}$. In this situation any extrapolation of the potential to the central region is found to be rather ambiguous. Fits of the differential cross section based on a Woods-Saxon parametrization of the optical potential usually yield values of $V_{0} \approx-(30 \div 80) \mathrm{MeV}$ for the central domain of nuclei [17]. However, it was shown [16] that values as deep as $-300 \mathrm{MeV}$ also yield reasonable results. In fact, even the deep potentials predicted by the RMFM [18] can not be excluded experimentally (for a further discussion see $[19,20]$ ).

The nucleon density corresponding to the strong-absorption radius may be estimated to be about $p_{0} / 10$ or less. One cannot expect that the mean-field approximation is valid in such dilute matter. The $t$-matrix approach should be more adequate in this situation, where an incident antiproton undergoes only a few (maybe one) collisions with peripheral target nucleons. This has indeed been demonstrated in $[21,22]$. The difference in the nucleon and antinucleon interaction with nuclei discussed above may explain the apparent violation [17] 
of the $G$-parity relation between their optical potentials. Indeed, the $G$-parity argument may be applied only to test nucleons and antinucleons propagating in the same external fields.

The applicability of the RMFM could be verified if the evidence for the predicted deep antinucleon potentials were found. The necessary conditions are realized potentially in high-energy heavy-ion collisions. In this case antinucleons may be produced in individual NN collisions or by the collective mechanism, described below, inside the dense baryonrich medium. Then the large difference in the mean potentials acting on the nucleons and antinucleons may lead to the substantial difference in the slope parameters of the proton and antiproton spectra which has been demonstrated in [23]. This effect is indeed observed in the AGS experiments [24].

\section{Spontaneous baryon-antibaryon pair creation}

It is interesting to see how the potentials behave at increasing baryon density $\rho \mathrm{v}$. From equation (17) it follows that the vector potential (12) changes linearly with $\rho \mathrm{v}$. The scalar potential (11) increases with $\rho \mathrm{v}$, but more slowly than the vector potential. In fact, the scalar potential tends to saturate at high baryon densities. Therefore the energy gap, $2 m_{\mathrm{B}}^{*}$, between all baryon and antibaryon states diminishes. The boundaries of the gap bend upwards with increasing baryon density. This is illustrated in figure 3.

At high enough density $\left(\rho_{V}=\rho_{c}\right)$ the upper edge of the Dirac sea raises above the lower edge of the upper continuum (in vacuum this corresponds to the energy $m_{\mathrm{B}}$ ). In other words, this means that the minimal antibaryon energy becomes lower than $-m_{\mathrm{B}}$. This situation in strong fields is well known as the Klein paradox [25]. Under such conditions the finite system becomes unstable with respect to the spontaneous baryon-antibaryon pair creation at the boundary, where the meson fields vary very rapidly. As a result, a strongly bound antibaryon appears in the dense nuclear medium accompanied by a low-energy baryon in vacuum. This process is indicated in figure 3 by the horizontal arrow, which shows the tunnelling transition of a baryon from the occupied lower continuum state into the empty upper continuum. The hole in the lower continuum is usually interpreted as the antiparticle.

The appearance of antibaryons in dense baryonic matter lowers the baryon density and, therefore, provides a negative feedback in the pair-creation process. Note that this is a consequence of the spatial separation of the pair. The instability is terminated when $\rho_{\mathrm{V}}$ decreases to $\rho_{\mathrm{c}}$. Many single-particle states become unstable at the critical density in the present case of an extended potential well. If the spontaneous decay were very fast compared to the compression and expansion time-scales in relativistic heavy-ion collisions, then the critical density would be the limiting baryon density, which could not be exceeded in a collision process. So if a baryon density higher than this critical density is generated in a certain region by some dynamical processes, it will drop down due to the creation of antibaryons (the baryons go outside) until it reaches the limiting value. In contrast, the spontaneous electron-positron pair production in a strong electric field generated by a nucleus of an overcritical charge $Z \geqslant 170[26,27]$ is associated with the diving of only one $\left(1 s_{1 / 2}\right)$ level into the negative energy sea. The phenomenon discussed here is similar to the "electron condensation' around a hypothetical 'supercharged nucleus' studied in [28]. However, in the case considered antibaryons are produced in the baryon-rich environment and, therefore, can easily annihilate while the baryons escape into the vacuum.

It is obvious that all baryonic states obeying the condition

$$
E_{\bar{B}}(p)=-U_{\mathrm{V} B}+\sqrt{\boldsymbol{p}^{2}+m_{\mathrm{B}}^{* 2}} \leqslant-m_{\mathrm{B}}
$$


Table 1. The critical baryon densities for spontaneous NN pair production.

\begin{tabular}{|c|c|c|c|c|c|c|c|c|c|}
\hline$C_{s}^{2}$ & $C_{\mathrm{v}}^{2}$ & $C_{3}$ & $C_{4}$ & $\frac{m_{\mathrm{N}}^{*}\left(\rho_{0}\right)}{m_{N}}$ & $\begin{array}{l}K\left(\rho_{0}\right) \\
(\mathrm{MeV})\end{array}$ & $\rho_{\mathrm{c}} / \rho_{0}$ & $\begin{array}{l}m_{\mathrm{N}}^{*}\left(\rho_{\mathrm{c}}\right) \\
(\mathrm{MeV})\end{array}$ & $\begin{array}{l}E_{\mathrm{c}}^{k_{\mathrm{p}}} \\
(\mathrm{GeV} N)\end{array}$ & Ref. \\
\hline 380.1 & 294.1 & 0 & 0 & 0.53 & 576.3 & 2.88 & 168.7 & 2.00 & [31] \\
\hline 358.2 & 274.5 & 0 & 0 & 0.54 & 544.4 & 3.09 & 169.6 & 2.58 & [30] \\
\hline 267.1 & 195.9 & 0 & 0 & 0.56 & 544.6 & 4.35 & 175.3 & 6.95 & [1] \\
\hline 261.0 & 209.9 & 0 & 0 & 0.64 & 300 & 4.21 & 184.7 & 6.44 & {$[32]^{4}$} \\
\hline 211.4 & 182.3 & 0 & 0 & 0.69 & 210 & 4.94 & 192.9 & 9.57 & {$[32]^{*}$} \\
\hline 289.8 & 199.4 & $5.48 \times 10^{-4}$ & $1.15 \times 10^{-3}$ & 0.65 & 400 & 4.42 & 213.8 & 7.31 & [33] \\
\hline 286.5 & 191.0 & $6.41 \times 10^{-4}$ & $2.00 \times 10^{-3}$ & 0.67 & 399.2 & 4.68 & 230.2 & 8.42 & [2] \\
\hline 233.2 & 132.5 & $3.29 \times 10^{-3}$ & $3.99 \times 10^{-3}$ & 0.75 & 300 & 7.04 & 279.9 & 21.4 & [33] \\
\hline 240.4 & 131.2 & $4.75 \times 10^{-3}$ & $6.19 \times 10^{-4}$ & 0.75 & 281.3 & 6.99 & 260.2 & 21.1 & [27] \\
\hline 240.4 & 131.2 & $4.75 \times 10^{-3}$ & $6.19 \times 10^{-4}$ & 0.75 & 281.3 & 6.33 & 147.3 & 16.9 & {$[27]^{b}$} \\
\hline 209.8 & 132.5 & $-2.45 \times 10^{-3}$ & $1.93 \times 10^{-2}$ & 0.75 & 400 & 7.40 & 342.3 & 23.8 & [33] \\
\hline 218.2 & 102.6 & $8.95 \times 10^{-3}$ & $3.69 \times 10^{-3}$ & 0.80 & 240.0 & 9.35 & 314.7 & 38.8 & [34] \\
\hline
\end{tabular}

a In this model, the expression for the vector potential contains a phenomenological term $-C_{\mathrm{d}} \rho_{B}^{1 / 3}$. The two versions given in the table correspond to the values $C_{\mathrm{d}}=0.183$ and 0.254 .

${ }^{b}$ In addition, $\Delta$-isobars are taken into account with a scalar coupling constant which is 1.31 times larger than that for the nucleon; the vector coupling constants are set to be equal (see also figure 1).

(i.e. $\left.E^{-}(-p) \geqslant m_{\mathrm{B}}\right)$ will be unstable. The total number of such states per unit volume is

$$
n=\frac{v_{B} p_{\max }^{3}}{3 \pi^{2}}
$$

where $p_{\max }$ is given by (18) with the equality sign:

$$
p_{\max }=\sqrt{\left(U_{\mathrm{V} B}-U_{\mathrm{SB}}\right)\left(U_{\mathrm{V} B}+U_{\mathrm{S} B}-2 m_{\mathrm{B}}\right)} .
$$

The critical baryon density $\rho_{\mathrm{c}}$ corresponds to $p_{\max }=0$. This leads to the equation

$$
U_{\mathrm{V} B}-m_{\mathrm{B}}^{*}=m_{\mathrm{B}}
$$

or

$$
U_{\mathrm{Y} B}+U_{\mathrm{SB}}=2 m_{\mathrm{B}}
$$

i.e. the instability occurs when the sum of the vector and scalar potentials exceeds the gap between positive- and negative-energy states in vacuum. This conclusion is valid for any baryon species $B$, irrespective of the origin of $\omega$ and $\sigma$ fields. If thess fields are generated by purely nucleonic matter, one can use for $\sigma$ and $\omega_{0}$ the explicit expressions obtained above. Combining equations (22) and (16) one arrives at the transcendental equation for the critical baryon (nucleon) density $\rho_{\mathrm{c}}^{(B)}$ for each baryon species $B$.

Figure 1 illustrates the geometrical determination of the critical density. The instability condition for the nucleons can be rewritten as $m_{\mathrm{N}}^{*}=U_{\mathrm{VN}}-m_{\mathrm{N}}$ where the RHS has a linear dependance on the baryon density. The critical density corresponds to the point where this line crosses the curve for $m_{\mathrm{N}}^{*}$. In figure 1 the calculation for the orignal parameter set of Walecka (solid lines) is compared to that of the parameter set NL075 (dotted lines), which gives a softer equation of state [29]. The critical point for the latter case is definitely at a higher density than that for the Walecka set, but the inclusion of $\Delta$-isobars (dash-dotted lines) can lower the critical density. It is assumed that $\Delta$ 's appear in the ground state when the nucleon chemical potential exceeds the difference of the $\Delta$ and $\mathrm{N}$ effective masses. 
The critical baryon density $\rho_{c}^{(N)}$ for spontaneous nucleon-antinucleon pair production is given in table 1 for several sets of model parameters used in [1,2,29-34]. We also show the nucleon effective mass at the critical density, as well as the nucleon effective mass and incompressibility modulus at normal nuclear density. Note that the parameter set of [29] leads to a very good description of finite nuclei. From the table, one can see that for the majority of the models the critical density $\rho_{c}^{(N)}$ lies in the range of $(3-7) \rho_{0}$ with an effective nucleon mass about $200 \mathrm{MeV}$. This density depends strongly on the effective mass $m_{\mathrm{N}}^{*}\left(\rho_{0}\right)$ and (via the Hugengoltz-van Hove theorem [33]) on the vector coupling $C_{V}$. It is clear that the effective mass will decrease more and that the baryon-antibaryon pair production will be enhanced at finite temperatures [10]. Many model calculations show that the required baryon densities and temperatures may well be reached in the course of relativistic nuclear collisions. For instance, in $[35,37,38]$ the density of shocked matter has been calculated using RMFM. Baryon densities of 3 to $5 \rho_{0}$ are predicted at bombarding energies of about $2 \mathrm{GeV} /$ nucleon. Calculations based on relativistic quantum molecular dynamics [39] lead to maximum baryon densities exceeding $6 \rho_{0}$ and $10 \rho_{0}$, respectively, in central collisions of $\mathrm{Si}+\mathrm{Au}$ at $14.5 \mathrm{GeV} / \mathrm{nucleon}$ and $\mathrm{Pb}+\mathrm{Pb}$ at $10 \mathrm{GeV} /$ nucleon. The average baryon densities close to, and in some cases above, the critical densities given in table 1 are also predicted by a two-fluid dynamical model [40].

Due to reduction of the baryon effective mass, the vacuum polarization effects, associated with the excitation of virtual baryon-antibaryon pairs, should be important in strong meson fields. As demonstrated in [38], these effects, at least partly, are taken into account by self-interaction terms in $V(\sigma)$. As one can see from table 1 , these terms shift the critical densities to higher values, compared with the linear version of the RMFM

It should be noted that the stopping regime in relativistic heavy-ion collision takes place only at energies of $\lesssim 10 \mathrm{GeV} /$ nucleon $[40,41]$. At higher bombarding energies the partial transparency effects become increasingly important. It is interesting that strong vector fields may be generated in this two-stream regime, too. Indeed, let us consider two interpenetrating nucleon flows of the same density. In its rest frame, each flow generates only the time-like component of the vector field $\omega_{0}$. In the equal velocity (EV) frame, where the flows move with velocities $v_{0}$ and $-v_{0}$ along the beam axis, the vector fields of the projectile $\left(\omega_{+}^{\mu}\right)$ and target $\left(\omega_{-}^{\mu}\right)$ flows can be written as

$$
\omega_{ \pm}^{\mu}=\left(\gamma \omega_{0}, 0,0, \pm \gamma v_{0} \omega_{0}\right)
$$

where $\gamma=\left(1-v_{0}^{2}\right)^{-1 / 2}$. In accordance with the superposition principle the net vector field in the overlap region of nucleon flows is

$$
\omega^{\mu}=\omega_{+}^{\mu}+\omega_{-}^{\mu}=\left(2 \gamma \omega_{0}, 0,0,0\right)
$$

Comparing (24) and (17) one may conclude that the factor $2 \gamma$ in the amplitude of the vector field in the case of interpenetrating nucleon flows is equivalent to the factor $\rho_{\mathrm{V}} / \rho_{0}$ in the case of compressed equilibrated matter. Bearing this in mind, one can easily calculate the critical bombarding energy (per nucleon in the lab frame) $E_{c}^{\text {kin }}=2 m_{\mathrm{N}}\left(\gamma_{\min }^{2}-1\right)$ for spontaneous $\mathrm{N} \bar{N}$ pair productiont. The results of this calculation performed for different model parameters are listed in table 1 . One can see that in most cases the values of $E_{c}^{\text {kin }}$ are rather low. At some choices of RMFM parameters $E_{c}^{\mathrm{kin}}$ is comparable with the threshold energy $5.6 \mathrm{GeV}$ for nucleon-antinucleon pair production in the individual nucleon-nucleon

if Here we consider the free-streaming limit, disreganding the mutual slowing down and compression of interpenetrating nucleon flows. 
collisions in vacuum. It is worthwhile noting here that this threshold energy should be lower by the factor $m_{\mathrm{N}}^{*} / m_{\mathrm{N}}$ due to the reduction of the effective mass in the medium.

However, these predictions should be regarded with some caution. It is well known that the RMFM predicts a linear energy dependence of the nucleon-nucleus optical potential, whereas the analysis of data on $\mathrm{pA}$ collisions show a saturation at energies $\gtrsim 0.3 \mathrm{GeV}$. As argued in [42] the exchange corrections to the mean-field approximation suppress the interaction of fast nucleons with the vector field of nuclear matter. Therefore, the simple formula (24) may overestimate the amplitude of the $\omega$-field in the case of interpenetrating nuclei moving with high enough relative energies.

It is interesting to estimate also the critical baryon density $\rho_{c}^{(\Lambda)}$ for spontaneous lambdaantilambda pair production. Our calculations show that $\rho_{\mathrm{c}}^{(\Lambda)}$ has reasonable values, say less than $7 \rho_{0}$, only if the lambda-meson coupling constants are close to the corresponding nucleon constants. As was shown in [43], experimental data on hypernuclei do not exclude such a possibility.

\section{Estimate of the spontaneous pair-production rate}

It may be shown that the antibaryon states with energy $E_{\bar{B}}<-m_{\mathrm{B}}$ are metastable at $\rho_{\mathrm{V}}>\rho_{c}^{(B)}$. To estimate the lifetime of these quasistationary states we assume that the nuclear matter is separated from vacuum by a plane surface of width $d$ and that the mesoninduced potentials change linearly in the surface region from their inner values $U_{\mathrm{V} B}, U_{\mathrm{SB}}$ to zero. Then, in close analogy with the Schwinger formula [46], the barrier penetration probability in a quasiclassical approximation is given by the expression

$P\left(E, p_{\mathrm{T}}\right)=\exp \left[-\pi d \frac{\left(m_{\mathrm{B}} U_{\mathrm{V} B}-E U_{\mathrm{S} B}\right)^{2}+p_{\mathrm{T}}^{2}\left(U_{\mathrm{V} B}^{2}-U_{\mathrm{S} B}^{2}\right)}{\left(U_{\mathrm{V} B}^{2}-U_{\mathrm{S} B}^{2}\right)^{3 / 2}}\right]$

where $E$ and $p_{\mathrm{T}}$ are the energy and the transverse (with respect to the surface) momentum of a baryon produced in the vacuum. These quantities are conserved in the course of the tunnelling process. They vary in the limits $\sqrt{m_{\mathrm{B}}^{2}+p_{\mathrm{T}}^{2}} \leqslant E \leqslant E_{\max }\left(p_{\mathrm{T}}\right)$ and $0 \leqslant p_{\mathrm{T}} \leqslant\left(p_{\mathrm{T}}\right)_{\max }$, where

$$
\begin{aligned}
& E_{\max }\left(p_{\mathrm{T}}\right)=U_{\mathrm{V} B}-\sqrt{p_{\mathrm{T}}^{2}+\left(m_{\mathrm{B}}-U_{\mathrm{S} B}\right)^{2}} \\
& \left(p_{\mathrm{T}}\right)_{\max }=\frac{1}{2 U_{\mathrm{V} B}} \sqrt{\left(U_{\mathrm{V} B}^{2}-U_{\mathrm{S} B}^{2}\right)\left[U_{\mathrm{V} B}^{2}-\left(2 m_{\mathrm{B}}-U_{\mathrm{S} B}\right)^{2}\right]} .
\end{aligned}
$$

We define the pair production rate as the number of pairs with the baryon energies between $E$ and $E+\mathrm{d} E$ and transverse momenta between $p_{\mathrm{T}}$ and $p_{\mathrm{T}}+\mathrm{d} p_{\mathrm{T}}$ produced per unit surface area and per unit time

$$
I\left(E, p_{\mathrm{T}}\right)=\frac{\mathrm{d} N_{\text {pair }}}{\mathrm{d} E \mathrm{~d}^{2} p_{\mathrm{T}} \mathrm{d} S \mathrm{~d} t} .
$$

This quantity may be calculated analytically only for selected simple potentials. We have not found the exact solutions for the combination of linear vector and scalar potentials in the literature. The case of the linear vector potential in a limited spatial domain was considered in [47]. We circumvent this problem by assuming that the rate (28) may be represented as

$$
I\left(E, p_{\mathrm{T}}\right)=I_{0}\left(E, p_{\mathrm{T}}\right) P\left(E, p_{\mathrm{T}}\right)
$$


where $P$ is the barrier penetration factor (25) which depends strongly on $d$, and $I_{0}$ is a smooth function of $d$. It may be calculated rather easily in the lowest approximation in $d / R$, i.e. for semi-infinite nuclear matter with a sharp boundary. Then we have [47]

$$
I_{0}\left(E, p_{\Upsilon}\right)=\frac{\nu_{B}}{(2 \pi \hbar)^{3}} \ln \mathcal{R}^{-1}
$$

where $\mathcal{R}$ is the reflection coefficient for the step-like potential. This coefficient is well known for the single vector or the scalar potential (see, for instance, [25]). We calculated it for the case of the combined vector and scalar step-like potentials of height $U_{S}$ and $U_{\mathrm{V}}$, respectively (for brevity here and below we drop index $B$ ). We arrived at the following result (see appendix):

$$
\mathcal{R}=\frac{m_{\mathrm{T}} m_{T}^{*} \cosh \left(y-y^{*}\right)+p_{\mathrm{T}}^{2}+m m^{*}}{m_{\mathrm{T}} m_{\mathrm{T}}^{*} \cosh \left(y+y^{*}\right)+p_{\mathrm{T}}^{2}+m m^{*}}
$$

where the internal $y^{*}$ and external $y$ rapidities are defined by expressions

$$
E=m_{\mathrm{T}} \cosh y \quad U-E=m_{\mathrm{T}}^{*} \cosh y^{*}
$$

while the internal $m_{\mathrm{T}}^{*}$ and external $m_{\Upsilon}$ transverse masses are

$$
m_{T}^{*}=\sqrt{m^{* 2}+p_{\mathrm{T}}^{2}} \quad m_{\mathrm{T}}=\sqrt{m^{2}+p_{\mathrm{T}}^{2}} .
$$

Here $m^{*}=m-U_{\mathrm{S}}$ is the internal effective mass and $m$ the baryon mass in vacuum. In the case of a pure vector potential $\left(U_{\mathrm{S}}=0\right)$ one arrives at the known result represented in a slightly unusual form

$$
\mathcal{R}=\frac{\cosh \left(y-y^{*}\right)+1}{\cosh \left(y+y^{*}\right)+1} .
$$

One can consider equation (29) as an interpolating formula which gives correct answers in the two limiting cases $d \rightarrow 0$ and $d \rightarrow \infty$ and, therefore, it may be used for rough estimates at any $d$.

It is easy to show that $\ln \mathcal{R}^{-1}$ vanishes at the kinematic boundary in the $\left(E, p_{\mathrm{T}}\right)$-plane defined by (26) $+(27)$ and that $\ln \mathcal{R}^{-1}$ has values of the order of unity, except for the regions adjacent to this boundary. Using (28)-(33) one can easily calculate the spectra and the yields of nucleons and antinucleons originating from the spontaneous $\mathrm{N} \overline{\mathrm{N}}$ production at various densities of nuclear matter. The $p_{\mathrm{T}}$-spectrum of produced baryons is obtained by integrating (28) over $E$. It drops approximately as $\exp \left(-p_{\mathrm{T}}^{2} / 2 m_{\mathrm{N}} T_{\text {eff }}\right)$ with an effective 'temperature' $T_{\text {eff }} \sim \hbar / 2 \pi d \sim 10-30 \mathrm{MeV}$ (at $d \sim 1-3 \mathrm{fm}$ ).

To get an idea about the magnitude of the pair-production effect we performed the calculations with the coupling parameters of [2]. The calculated rates of the NN pair production per unit surface area and unit time, $\mathrm{d} N_{\text {pair }} / \mathrm{d} S \mathrm{~d} t$, are given in table 2. As one can see from this table, the results are very sensitive to the density of compressed matter and to the width of the surface region $d$. In the same table we also present the total numbers of pairs $N_{\text {pair }}$ assuming that the compressed region is formed in a central collision of equal nuclei of mass $A$. For the surface area we take $2 \pi r_{0}^{2} A^{2 / 3}\left(r_{0}=1.1 \mathrm{fm}\right)$. We also assume that the lifetime of the compressed state is equal to the nuclei passage time $\tau_{\text {pass }}=2 r_{0} A^{1 / 3} / v_{0} \gamma$ in the $\mathrm{EV}$ frame. This estimate agrees well with the time-scale 
Table 2. Yield of NN pairs spontaneously produced in a slab of compressed nuclear matter.

\begin{tabular}{|c|c|c|c|c|}
\hline \multirow[b]{2}{*}{$\frac{\rho}{\rho_{0}}$} & \multicolumn{2}{|c|}{$d=1 \mathrm{fm}$} & \multicolumn{2}{|c|}{$d=2 \mathrm{fm}$} \\
\hline & $\frac{d N_{p a j r}}{d S d r}\left(c \mathrm{fm}^{-3}\right.$ & $\frac{N_{\text {pulr }}}{X} \gamma$ & $\frac{d N_{\text {pair }}}{d s d r}\left(c \mathrm{fm}^{-3}\right.$ & $\frac{N_{\text {pair }}}{\hbar} \gamma$ \\
\hline$\overline{5}$ & $3.76 \times 10^{-5}$ & $6.29 \times 10^{-4}$ & $1.34 \times 10^{-6}$ & $2.23 \times 10^{-5}$ \\
\hline 6 & $3.98 \times 10^{-3}$ & $6.66 \times 10^{-2}$ & $3.57 \times 10^{-4}$ & $5.97 \times 10^{-3}$ \\
\hline 7 & $2.68 \times 10^{-2}$ & $4.48 \times 10^{-1}$ & $3.99 \times 10^{-3}$ & $6.68 \times 10^{-2}$ \\
\hline
\end{tabular}

extracted from dynamical calculations $[39,40]$. The yield of spontaneous antinucleons per participant nucleon is estimated on the level of $10^{-5}-10^{-4}$ for the collision energies about $10 \mathrm{GeV} /$ nucleon $(\gamma \approx 3)$, assuming $\rho / \rho_{0} \approx 5$. The antiproton yields of this order of magnitude have been measured in AGS experiments at Brookhaven [24] (see also [48]).

The possibility of anti-matter cluster production is of great interest. The mechanism considered above may lead to an enhanced yield of such objects as $\overline{\mathrm{d}}, \bar{\alpha}$. The reason is that in large enough systems many nucleon states become unstable simultaneously. The correlated decay of this states will lead to the enhanced formation of multi-antinucleon clusters. For instance, if one considers $\alpha$ and $\bar{\alpha}$ as elementary particles with coupling constants and mass equal to corresponding nucleon values multiplied by a factor four, then the ratio of $\bar{\alpha}$ and $\overline{\mathrm{p}}$ yields is approximately proportional to $P^{3}(25)$. At the same parameters as before, this gives an extra factor of the order of $10^{-6}-10^{-9}$ for the $\bar{\alpha}$ yield, compared with the predicted yield of antinucleons. This abundance is small but measurable [49] and we would strongly recommend such measurements.

\section{Conclusions}

The RMFM predicts a strongly attractive interaction of antibaryons with the surrounding baryon-rich matter. This may lead to interesting observable effects aiready at moderate densities, of the order of $\rho_{0}$. For instance, antibaryons produced in the interior of the nucleus or inside the nuclear fireball have a chance to leave the system only if their kinctic energy is high enough, i.e. $E^{\text {kin }}>m_{\mathrm{B}}+\left|U_{\bar{B}}\right|$. Slow antibaryons will be trapped in the deep potential and will most probably annihilate inside the system. This situation is discussed in [10] where a system of baryons, antibaryons and mesons in thermal equilibrium is considered. As was shown in [23] the different shapes of the nucleon and antinucleon spectra may be explained by strong mean fields, repulsive for nucleons and attractive for antinucleons, of the same order of magnitude as predicted by the Walecka model. On the other hand, the cascade models which do not take into account mean felds are not able to explain the data. The importance of mean fields and annihilation effects for the antiproton yields in heavy-ion collisions was emphasized recently within the framework of the RBUU model [51].

Other even more dramatic phenomena may also occur in the course of high-energy heavy-ion collisions where strong space and time variations of meson fields are expected; it was demonstrated above that the baryonic states of the negative Dirac sea may play an important role in such a situation. In the present paper we consider the possibility of spontaneous baryon-antibaryon pair creation at the boundary between the dense baryonic matter and the vacuum. Dynamical calculations which take into account the vacuum rearrangement effects in time-dependent meson fields are required to quantify these predictions. The baryon-antibaryon pair production induced by the strong time-dependent 
fields might be more effective than the spontaneous one, in analogy to the electron-positron case. These collective mechanisms, as well as the themal excitation of the low continuum states, potentially lead to a considerable enhancement of antibaryon and antihyperon yields compared to the predictions of the models based on the free nucleon-nucleon collisions. Antimatter cluster production may also become feasible on a measurable level. We think that many observable signals proposed for the quark-gluon plasma, in particular enhanced antihyperon yields, may be interpreted in the purely hadronic scenario, if the reduction of hadron masses in dense matter is taken into account [10].

The annihilation of antibaryons in the surrounding matter must be included in a refined realistic calculation [23,52]. The antibaryon annihilation depends drastically on the inmedium annihilation cross section. A large variety of annihilation channels are potentially suppressed due to the decreased baryon effective masses. Therefore the annihilation cross section should go down with decreasing $m_{\mathrm{B}}^{*}$. In the extreme case when $m_{\mathrm{B}}^{*}<m_{\pi}$ pionic final states are forbidden and the strong annihilation cross section vanishes. For the same reason the threshold energy for $B \bar{B}$ pair production in individual NN collisions in matter should be considerably lower than in vacuum. One additional important question to be solved is how the 'in medium' baryons and antibaryons with reduced effective masses go on the vacuum mass shell.

\section{Acknowledgments}

The authors are grateful to A Schäfer and P-G Reinhard for fruitful discussions. We also thank $\mathrm{H}$ Sorge and $\mathrm{H}$ Feldmeier for useful remarks. One of us (HS) likes to thank J Boguta for exciting discussions concerning the discussed critical phenomenon. Two of us (INM and LMS) are grateful to the Institut für Theoretische Physik der J W Goethe Universitat for kind hospitality and financial support.

\section{Appendix. The reflection coefficient for supercritical scalar and vector potentials}

Below, the formula (31) for the reflection coefficient is derived. We consider the case of semi-infinite nuclear matter of a constant density at $z>0$. In the local approximation the interaction of baryons with this matter can be described by the combination of the step-like scalar $U_{\mathrm{S}} \Theta(z)$ and vector $U \Theta(z)$ potentials, where $\Theta(z)=\frac{1}{2}(1+\operatorname{sign} z)$. Hereafter, the index $B$ is omitted for brevity. The solution of the Dirac equation can be obtained by generalizing the procedure suggested in $[47,53]$. The wavefunctions in regions $\mathrm{I}(z<0)$ and II $(z>0)$ can be represented as

$$
\Psi_{\mathrm{I}, \mathrm{I}}(t, r)=\mathrm{e}^{-\mathrm{i} E t+\mathrm{i} p_{\mathrm{T}} \boldsymbol{r}_{\perp}} \psi_{\mathrm{I}, \mathrm{I}}(z) .
$$

Here $E$ and $p_{\mathrm{T}}$ are the energy and transverse momentum of a baryon in vacuum. Substituting (A1) into the Dirac equation (4) we get the equations for spinors $\psi_{1, I I}(z)$ :

$$
\begin{aligned}
& \left(\gamma_{\mu} p_{\mathrm{I}}^{\mu}-m\right) \psi_{1}(z)=0 \\
& \left(\gamma_{\mu} p_{\mathrm{II}}^{\mu}-m^{*}\right) \psi_{\mathrm{I}}(z)=0
\end{aligned}
$$

where

$$
\begin{aligned}
& p_{\mathrm{I}}^{\mu}=\left(E, p_{\mathrm{T}},-\mathrm{i} \partial_{z}\right) \\
& p_{\mathrm{II}}^{\mu}=\left(E-U, p_{\mathrm{T}},-\mathrm{i} \partial_{z}\right) \\
& m^{*}=m-U_{\mathrm{S}} .
\end{aligned}
$$


The continuity condition at $z=0$ is

$$
\psi_{1}(-0)=\psi_{\Pi}(+0)
$$

In our case of a spin-independent interaction it is useful to represent $\psi_{\mathbb{D}}(z)$ as

$$
\psi_{\Pi}(z)=\left(\gamma_{\mu} p_{\mathrm{\Pi}}^{\mu}+m^{*}\right)\left\{\left(\begin{array}{l}
1 \\
0 \\
1 \\
0
\end{array}\right) f_{\mathrm{\Pi}}^{(1)}(z)+\left(\begin{array}{c}
0 \\
1 \\
0 \\
-1
\end{array}\right) f_{\Pi}^{(2)}(z)\right\}
$$

Hereafter we use the standard representation [54] of Dirac matrices. The equations for scalar functions $f_{\square}^{(\alpha)}(\alpha=1,2)$ are obtained by substituting (A5) into (A3). Then we have

$$
\left[\partial_{z}^{2}+\left(E-U_{\mathrm{V}}\right)^{2}-m_{\mathrm{T}}^{* 2}\right] f_{\mathrm{I}}^{(\alpha)}=0
$$

where $\alpha=1,2$ and $m_{\mathrm{T}}^{*}$ is defined in (33).

The analogous representation for $\psi_{\mathrm{I}}(z)$ is given by (A5) with the replacement $\mathrm{II} \rightarrow \mathrm{L}$, $m^{*} \rightarrow m$. Using (A2) we get the equations for $f_{\mathrm{I}}^{(\alpha)}(\alpha=1,2)$ :

$$
\left[\partial_{z}^{2}+E^{2}-m_{\mathrm{T}}^{2}\right] f_{\mathrm{I}}^{(\alpha)}=0
$$

where $m_{\mathrm{T}}$ is defined in (33).

The general solutions of (A6), (A7) can be written as

$$
\begin{aligned}
& f_{\mathrm{I}}^{(\alpha)}=a_{\alpha} \mathrm{e}^{\mathrm{i} k_{1} z}+b_{\alpha} \mathrm{e}^{-\mathrm{i} k_{1} z} \\
& f_{\Pi}^{(\alpha)}=c_{\alpha} \mathrm{e}^{\mathrm{i} k_{\Pi^{z}}}+d_{\alpha} \mathrm{e}^{-\mathrm{i} k_{\mathrm{\Pi}^{z}}}
\end{aligned}
$$

where

$$
\begin{aligned}
& k_{\mathrm{I}}=\sqrt{E^{2}-m_{\mathrm{T}}^{2}}=m_{\mathrm{T}} \sinh y \\
& k_{\mathrm{I}}=\sqrt{\left(E-U_{\mathrm{V}}\right)^{2}-m_{\mathrm{T}}^{* 2}}=m_{\mathrm{T}}^{*} \sinh y^{*} .
\end{aligned}
$$

Here the definitions of (32) were used. The coefficients $a_{\alpha}, b_{\alpha}, c_{\alpha}, d_{\alpha}$ should be determined from the boundary conditions.

We are interested in the case when the spontaneous production of a $B \bar{B}$-pair is possible at given $E$ and $p_{\mathrm{T}}$. Then $U_{\mathrm{Y}}>E+m_{\mathrm{T}}^{*}(26)$ and $k_{\mathrm{n}}$ is real. In this case $\psi_{\mathrm{I}}$ and $\psi_{\mathrm{II}}$ describe a propagation of a baryon and an antibaryon, respectively. Since by definition there is no reflected wave in the region $z>0$, we take $d_{\alpha}=0$ in (A8). From (A4) one gets four equations for coefficients $a_{\alpha}, b_{\alpha}, c_{\alpha}(\alpha=1,2)$. Then after eliminating $c_{1,2}$ we obtain the set of equations relating $a_{\alpha}$ and $b_{\alpha}$ :

$$
\left(\begin{array}{cc}
A(y) & p-B(y) \\
-p_{+} B(y) & A(y)
\end{array}\right)\left(\begin{array}{l}
b_{1} \\
b_{2}
\end{array}\right)+\left(\begin{array}{cc}
A(-y) & p_{-} B(-y) \\
-p_{+} B(-y) & A(-y)
\end{array}\right)\left(\begin{array}{l}
a_{1} \\
a_{2}
\end{array}\right)=\left(\begin{array}{l}
0 \\
0
\end{array}\right)
$$

where $p_{ \pm}=p_{x} \pm \mathrm{i} p_{y}$ and

$$
\begin{aligned}
& A(y)=m m_{\mathrm{T}}^{*} \mathrm{e}^{y^{*}}+m^{*} m_{\mathrm{T}} \mathrm{e}^{y} \\
& B(y)=m_{\mathrm{T}}^{*} \mathrm{e}^{y^{*}}+m_{\mathrm{T}} \mathrm{e}^{y} .
\end{aligned}
$$


The longitudinal component of the current in the region $I$ is

$$
\begin{gathered}
J_{\mathrm{I}}=\bar{\psi}_{\mathrm{I}}(z) \gamma^{3} \psi_{\mathrm{I}}(z)=2 \sum_{\alpha=1,2}\left[\left|m_{\mathrm{T}} f_{\mathrm{I}}^{(\alpha)}\right|^{2}-\left|\left(E+\mathrm{i} \partial_{z}\right) f_{\mathrm{I}}^{(\alpha)}\right|^{2}\right] \\
=4 k_{\mathrm{I}} \sum_{\alpha=1,2}\left[\left(E-k_{\mathrm{I}}\right)\left|a_{\alpha}\right|^{2}-\left(E+k_{\mathrm{I}}\right)\left|b_{\alpha}\right|^{2}\right] .
\end{gathered}
$$

The first and second term in the last equality in (A14) correspond, respectively, to the incoming $\left(J_{1}^{\text {in }}\right)$ and reflected $\left(J_{1}^{\text {ref }}\right)$ components of the total current. Using (A11) we obtain the expression for the reflection coefficient

$$
\mathcal{R}=\left|\frac{J_{1}^{\text {ref }}}{J_{1}^{\text {in }}}\right|=\frac{E+k_{\mathrm{I}}}{E-k_{\mathrm{I}}} \frac{\left|b_{1}\right|^{2}+\left|b_{2}\right|^{2}}{\left|a_{1}\right|^{2}+\left|a_{2}\right|^{2}} .
$$

Solving (A11) one can express $b_{\alpha}$ in terms of $a_{\alpha}$. Then we get the formula

$$
\mathcal{R}=\mathrm{e}^{2 y} \frac{B^{2}(-y)+p_{\mathrm{T}}^{2} A^{2}(-y)}{B^{2}(y)+p_{\mathrm{T}}^{2} A^{2}(y)} .
$$

As one should expect, $\mathcal{R}$ is independent of $a_{\alpha}$. Substituting (A12), (A13) into (A15) we arrive at the final result (31).

\section{References}

[1] Serot B D and Walecka J D 1985 Adv. Nucl. Phys. vol 16 (New York: Plenam)

[2] Reinhard P G 1989 Rep. Prog. Phys. 52439

[3] Theis J, Graebner G. Buchwald B, Marułn J A, Greiner W, Stöcker H and Polonyi J 1983 Phys. Rev. D 28 2286

[4] Bartke J (NA35 collaboration) 1990 Z. Phys. C48 191

[5] Abatzis S (WA85 collaboration) 1991 Phys. Lett. 270B 123

[6] Koch P and Rafelski J 1985 Nucl. Phys. A 444678

[7] Koch P, Müller B and Rafelski J 1986 Phys. Rep. 142167

[8] Mishustin IN 1990 Sov. J. Nucl. Phys. 521135

[9] Mishustin I N, Satarov L M, Schaffner J, Stöcker H and Greiner W 1991 Proc. Int. Conf. on High Energy Physics Problems (Dubna 1990) ed A M Baldin, V V Burov and L P Kaptari (Singapore: World Scientific) p 614

[10] Schaffner J, Mishustin I N, Satarov L M, Stöcker H and Greiner W 1991 Z. Phys. A 34147

[11] Boguta J and Bodmer A R 1977 Nucl. Phys. A 292414

[12] Boguta J and Stöcker H 1983 Phys. Lett. L20B 289

[13] Duerr H-P and Teller E 1956 Phys. Rev. 101494

[14] Shapiro I S 1978 Phys. Rep. C 35129

[15] Auerbach N, Goldhaber A S and Miller L D 1986 Phys. Lett. 182B 221

[16] Kubo K-I, Toki H and Igarashi M 1985 Nucl. Phys. A 435708

[17] Janouin S et al 1986 Nucl. Phys. A 451541

[18] Bouyssy A and Marcos S 1982 Phys. Lett. 114B 387

[19] Ingemarson A et al 1986 Nucl. Phys. A 454475

[20] Friedman E and Lichtenstadt J 1986 Nucl. Phys. A 455573

[21] Clark B C et al 1983 Phys. Rev. Lett. 501644

[22] Garreta D et al 1984 Phys. Lett. 149B 64

[23] Koch V, Brown G and Ko C M 1991 Phys. Lett. 265B 29

[24] Costales J (E802 collaboration) 1990 Talk presented at the Workshop on Heavy Ion Physics at AGS (BNL, Upton, 1990)

[25] Greiner W, Müller B and Rafelski J 1985 Quantum Electrodynamics of Strong Fields (Berlin: Springer) 
[26] Picper W and Greiner W 1969 Z. Phys. A 218327

[27] Popov V S 1970 Sov. J. Nucl. Phys. 12429

[28] Migdal A B 1978 Fermions and bosons in strong fields (Nauka: Moscow)

[29] Fink J, Blum V, Reinhard P-G, Maruhn J A and Greiner W 1989 Phys, Lett. 218B 277: 2233123

[30] Horowitz C J and Serot B D 1981 Nucl. Phys. A 368303

[31] Lee S J, Fink J, Balantakin A B, Strayer M R, Umar A S, Reinhard P-G, Manhn J A and Greiner W 1986 Phys. Rev. Lett. 591171

[32] Gorenstein M 1, Rischke D H, Bugaev K A, Stöcker H and Greiner W 1989 UFTP preprint $239 / 1989$ (Frankfurt-am-Main)

[33] Waldhauser B M, Maruhn J A, Stöcker H and Greiner W 1988 Phys. Rev. C 381003

[34] Glendenning N K 1987 Z. Phys. A 327295

[35] Stöcker H, Greiner W and Scheid W I978 Z. Phys. A 286121

[36] Stöcker H, Gyulassy M and Boguta J 1981 Phys. Lett. 103B 269

Waldhauser B M, Maruhn J A, Stöcker H and Greiner W 1987 Z. Phys. A 32819

[37] Glendenning N K 1988 Phys. Rev. C 371442

[38] Schōnhofen M S, Cubero M, Gering M, Sambataro M, Feldmeier H and Nörenberg W 1989 Nucl. Phys. A 504875

[39] von Keitz A, Berenguer M, Sorge H, Mattiello R, Stöcker H and Greiner W 1990 Proc. Int. Workshop on Gross Properties of Nuclei and Nuclear Exitations XVII (Hirschegg, 1990) (GSI, Darmstadt) p 80; 1990 Phys. Lett. 243B 7

[40] Mishustin I N, Russkikh V N and Satarov L M 1989 Nucl. Phys. A 494 595; 1991 Sov. J. Nucl. Phys. 54 429

[41] Nagamiya S 1988 Nucl. Phys. A 488 3c

[42] Blättel B, Koch V, Cassing W and Mosel U 1990 Proc. Int. Workshop on Gross Properties of Nuclei and Nuclear Exitations XVIII (Hirschegg, 1990) (GSI, Darmstadt) p 263

[43] Rufa M, Stöcker H, Maruhs J A, Reinhard P.G and Greiner W 1987 J. Phys. G: Nucl. Phys. 13 143

[44] Rufa M 1989 Doctoral Thesis, GSI-Report 89-19

Rufa M, Schaffner J, Maruhn J A, Stöcker H and Greiner W, Reinhard P-G 1990 Phys. Rev, C 422469

[45] Schaffiner J, Greiner W and Stöcker H 1992 Phys. Rev. C 46322

[46] Schwinger J 1951 Phys. Rev. 82664

[47] Wang R-C and Wong C-Y 1988 Phys. Rev. D 38348

[48] Dover C B 1990 Talk presented at the Workshop on Heavy Ion Physics at AGS (BNL, Upton, 1990) Braun-Munzinger $P$ (Private communication)

[49] Crawford $J$ et al 1990 Search for New Particles and investigation of Hadronization in Nucleus-Nucleus Collisions at the AGS AGS proposal

[50] Sandweiss J et al 1990 Production of Rare Composite Objects in Relativistic Heavy Ion Collisions BNL Proposal 864, 1990

[51] Cassing W, Lang A, Teis S and Weber K 1992 Proc. Int. Workshop on Dynamical Fluchuations and Correlations in Nuclear Collisions (Aussois, 1992) (Nucl. Phys.) A 545 121)

[52] Jahns A, Sorge H, Stöcker H and Greiner W 1991 UFTP preprint 285/1991; 1992 Phys. Rey. Lett. 682895

[53] Nikishov A I 1970 Nucl. Phys. B 21346

[54] Bjorken J D and Drell S D 1964 Relativistic quantum mechanics (New York: McGraw-Hill) 